\title{
Faunistic notes on the Mesochorinae (Hymenoptera: Ichneumonidae) of Russian Fennoscandia, with description of a new Mesochorus species from Karelia
}

\section{Фаунистические заметки о Mesochorinae (Hymenoptera: Ichneumonidae) российской Фенноскандии с описанием нового вида Mesochorus из Карелии}

\author{
Matthias Riedel ${ }^{1}$, Andrei Humala ${ }^{2^{*}}$ \\ Маттиас Ридель', Андрей Хумала ${ }^{2 *}$
}

\footnotetext{
${ }^{1}$ Bärenbad Str. 11, 82487 Oberammergau, Germany. E-mail: mamaflo.riedel@t-online.de

${ }^{2}$ Forest Research Institute, Russian Academy of Sciences, Pushkinskaya St. 11, Petrozavodsk 185910, Russia. E-mail: andrei.humala@krc.karelia.ru ${ }^{2}$ Институт леса Карельского научного центра Российской академии наук, ул. Пушкинская 11, Петрозаводск 185910 , Россия.
}

KEY WORDS: Mesochorinae, ichneumon wasps, fauna, NW Russia, new species.

КЛЮЧЕВЫЕ СЛОВА: Mesochorinae, наездники-ихневмониды, фауна, С3 Россия, новый вид.

ABSTRACT. A list of 40 species of Mesochorinae (Hymenoptera, Ichneumonidae) resulting from study of the material collected in the Karelia and Murmansk Areas as well as in some adjacent territories, is given. Among those, 15 species, namely, Astiphromma aggressor (Fabricius, 1804), A. nigrocoxatum (Strobl, 1904), A. tenuicorne (Thomson, 1886), A. uliginosum Schwenke, 1999, Mesochorus abraxator Schwenke, 1999, M. boreomontanus Schwenke, 1999, M. cacuminis Schwenke, 1999, M. cinctus Schwenke, 1999, M. eichhorni Schwenke, 1999, M. hortensis Schwenke, 1999, M. lacassus Schwenke, 1999, M. lapponicus Thomson, 1885, M. scandinavicus Schwenke, 1999, M. skaneus Schwenke, 1999, M. tenuiscapus Thomson, 1886, are reported for the territory of Russia for the first time. Mesochorus horstmanni Schwenke, 1999 is new for the fauna of Finland. Mesochorus tetricoides Riedel sp.n. from Kivach Nature reserve is also described and illustrated; species status of Mesochorus acutus Schwenke, 1999 stat.rev. is resurrected.

РЕЗЮМЕ. Составлен список 40 видов Mesochorinae (Hymenoptera, Ichneumonidae), полученный в результате обработки материалов из Карелии и Мурманской области, а также некоторых прилежащих территорий. Из них 15 видов, а именно, Astiphromma aggressor (Fabricius, 1804), A. nigrocoxatum (Strobl, 1904), A. tenuicorne (Thomson, 1886), A. uliginosum Schwenke, 1999, Mesochorus abraxator Schwenke, 1999, M. boreomontanus Schwenke, 1999, M. cacuminis Schwenke, 1999, M. cinctus Schwenke, 1999, M. eichhorni Schwenke, 1999, M. hortensis Schwenke, 1999, M. lacassus Schwenke, 1999, M. lapponicus Thomson, 1885, M. scandinavicus Schwenke,

* - Corresponding author
1999, M. skaneus Schwenke, 1999, M. tenuiscapus Thomson, 1886, впервые приводятся для российской территории. Mesochorus horstmanni Schwenke, 1999 является новым видом для фауны Финляндии. Из заповедника «Кивач» также описан Mesochorus tetricoides Riedel sp.n.; восстановлен видовой статус Mesochorus acutus Schwenke, 1999 stat.rev.

\section{Introduction}

The Republic of Karelia, as well as the Murmansk Area and Karelian Isthmus, belong to Eastern Fennoscandia and are situated in the North Western Russia. These territories are the least studied in terms of their insect fauna in Fennoscandia in general. Specifically, a thorough research of this kind is conducted in this territory only since the 1980s. This is also true for one of the largest insect families, i.e. ichneumon wasps. For example, more than 2600 species of this group are known for Finland [Söderman et al., 2010], a country with a similar territory and natural conditions. On the contrary, the Russian part of Fennoscandia is still insufficiently studied.

Members of the ichneumonid subfamily Mesochorinae were difficult to identify for a long time, and they were poorly studied until recently due to absence of adequate taxonomic treatment and reliable identification keys. European species of the genus Astiphromma Förster, 1869 have been recently revised [Riedel, 2015]. The present paper is the second one dealing with the subfamily Mesochorinae of Northwestern Russia. The first paper, with 33 listed species, has been recently published [Riedel, Humala, 2016], and a short review of this group can be found in this paper as well. The new faunistic reports deal mainly with Mesochorus Gravenhorst, 1829 species. 


\section{Material and methods}

All materials used in the present study were collected by the second author in different localities of Karelia, the Murmansk Area and some adjacent territories by sweep netting and use of various traps (Malaise, yellow pan and light ones). The materials studied are stored in collections of both authors. The type of the new species is deposited in the Zoologische Staatssammlung München (ZSM, Munich, Germany). The division of Fennoscandia into biogeographic provinces and corresponding abbreviations follow the proposals by Heikinheimo and Raatikainen [1971], with minor changes regarding the Russian territory introduced by Kravchenko and Kuznetsov [2001]. Latin names and general distributions of different species are given according to the latest version of World Ichneumonoidea Catalogue [Yu et al. 2016].

\section{Species list}

Genus Astiphromma Förster, 1869

Astiphromma aggressor (Fabricius, 1804)

MATERIAL. Karelia, Kon: Malaya Gomsel'ga, $1 \mathrm{~km}$ E, $62.066^{\circ} \mathrm{N} 33.98^{\circ} \mathrm{E}$, cutting area, $1+6$. VI.2013, leg. A. Humala.

DISTRIBUTION. Western Palaearctic region, new for Russia.

\section{Astiphromma anale (Holmgren, 1860)}

MATERIAL, Karelia, Kpor: Ladozero, $63.580^{\circ} \mathrm{N} 35.884^{\circ} \mathrm{E}$ spruce forest, yellow pan trap, 1우 26-29.VI.2010, leg. A. Humala; Karelia, Kk: Gridino $2 \mathrm{~km} \mathrm{SW}$, spruce forest, $65.9081^{\circ} \mathrm{N} 34.6304^{\circ} \mathrm{E}$, Malaise trap, 2 O90 4.VII.-9.VIII.2007, leg. A. Humala; Murmansk Area, Lps: Pasvik Nature reserve, Kalkupya mt., $69.2870^{\circ} \mathrm{N}$ 29.3554 ${ }^{\circ}$ E, Malaise trap 7.VI.-4.VII.2007, $20^{7} 0^{7}$, 4-30.VII.2007, $30^{3} \sigma^{7}$ leg. A. Humala; Murmansk Area, Lps: Pasvik Nature reserve, Varlam i., $69.1376^{\circ} \mathrm{N} 29.2616^{\circ} \mathrm{E}$, Malaise trap, 5 웅 $80^{7} 0^{7}$ 6.VI.10.VII.2007, $10^{7}$ 10.VII.-3.VIII.2007, leg. A. Humala; Murmansk Area, Lps: Pasvik Nature reserve, Menikkayoki, $69.3737^{\circ} \mathrm{N}$ $29.8824^{\circ} \mathrm{E}$, Malaise trap, $10^{7}$ 5.VI.-6.VII.2007, leg. A. Humala.

DISTRIBUTION. Known from Russia, including Karelia and Murmansk Area [Riedel, Humala, 2016].

\section{Astiphromma flavoventrale Riedel, 2015}

MATERIAL Murmansk Area, Lps: Pasvik Nature reserve, Menikkayoki, $69.3737^{\circ} \mathrm{N} 29.8824^{\circ} \mathrm{E}$, Malaise trap, $60^{7} \mathrm{O}^{7}$ 5.VI.6.VII.2007, 1우 6.VII.-14.VIII.2007, leg. A. Humala.

DISTRIBUTION. Known from Russia (Murmansk Area) [Riedel, Humala, 2016].

\section{Astiphromma nigrocoxatum (Strobl, 1904)}

MATERIAL Karelia, Kon: Kivach Nature reserve, $62.2646^{\circ} \mathrm{N}$ $33.9798^{\circ} \mathrm{E}$, aspen forest, Malaise trap, $1+27-29$. VI.1989, leg. A Humala; Karelia, Kon: Kizhi skerries, Malyi Lelikovskyi i., $61.98^{\circ}$ N, 35.16 E, meadows, 1.VII.2017, leg. A. Humala. Russia.

DISTRIBUTION. Western Palaearctic region, new for

Astiphromma splenium (Curtis, 1833)

MATERIAL. Karelia, Kl: Ristolahti surr., 9 km NE Sikopohja, $61.72^{\circ} \mathrm{N} 30.15^{\circ} \mathrm{E}, 1$ 9 7 VII.2005, leg. A. Humala; Karelia, Kon: Kivach Nature reserve, $62.2646^{\circ} \mathrm{N} 33.9798^{\circ} \mathrm{E}$, aspen forest, Malaise trap, 1 24.VIII.-25.IX.1989, leg. A. Humala; Karelia, Kon Belaya Gora, $62.583^{\circ} \mathrm{N} 33.948^{\circ} \mathrm{E}$, pine forest, $1 \mathrm{O}^{7} 16 . \mathrm{VII} .2002$, leg. A. Humala; Karelia, $K p$ : Prirechnyi $5 \mathrm{~km} \mathrm{~W}$, Cherga r., $61.792^{\circ} \mathrm{N}$ $37.510^{\circ} \mathrm{E}$, mixed forest, $10^{7} 25$. VI.2009, leg. A. Humala.
DISTRIBUTION. Known from Russia including Karelia [Riedel, Humala, 2016].

\section{Astiphromma striatum (Brischke, 1880)}

$=$ A. mandibulare Thomson, 1886

MATERIAL. Karelia, $K l$ : Kilpola i., $61.20^{\circ} \mathrm{N} 29.98^{\circ} \mathrm{E}$, mire, 1 우 16.VI.2011, leg. A. Humala; Karelia, Kl: $3 \mathrm{~km}$ E Lahdenpohja, $61.53^{\circ} \mathrm{N} 30.26^{\circ} \mathrm{E}, 19$ 15.VI.2015, leg. A. Humala; Karelia, Kol: Petrozavodsk-Lososinnoe, $61.7199^{\circ} \mathrm{E} 34.2186^{\circ} \mathrm{E}$, cutting area, plot 8, yellow pan trap, 1ㅇ 28-31.V.2013, leg. A. Humala.

DISTRIBUTION. Palaearctic region, known from Russia [Roman, 1931, Riedel, 2015] including Karelia [Riedel, Humala, 2016].

Astiphromma tenuicorne (Thomson, 1886)

MATERIAL. Karelia, Kon: Kivach Nature reserve, $62.2646^{\circ} \mathrm{N}$ $33.9798^{\circ} \mathrm{E}$, aspen forest, Malaise trap, 19 13-14.VII.1989, 19 27.VII.-3.VIII.1989, leg. A. Humala; Murmansk Area, Lps: Pasvik Nature reserve, Menikkayoki, $69.3737^{\circ} \mathrm{N} 29.8824^{\circ} \mathrm{E}$, Malaise trap, 1오 5.VI-6.VII.2007, leg. A. Humala.

DISTRIBUTION. Western Palaearctic region, new for Russia.

Astiphromma uliginosum Schwenke, 1999

MATERIAL. Murmansk Area, Lps: Pasvik Nature reserve, Varlam i., $69.1376^{\circ} \mathrm{N} 29.2616^{\circ} \mathrm{E}$, Malaise trap, 1 ㅇ 6.VI.10.VII.2007, leg. A. Humala.

DISTRIBUTION. Western Palaearctic region, new for Russia.

Astiphromma varipes (Holmgren, 1860)

MATERIAL. Karelia, Kon: Kivach Nature reserve, $62.2517^{\circ} \mathrm{N}$ $34.0070^{\circ} \mathrm{E}$, pine forest, Malaise trap, 1 우 5-9.VII.1990, leg. A. Humala.

DISTRIBUTION. Western Palaearctic region, known for Russia (Hellén, 1942), new for Karelia.

Genus Mesochorus Gravenhorst, 1829

Mesochorus abraxator Schwenke, 1999

MATERIAL. Murmansk Area, Lps: Pasvik Nature Reserve, Kalkupya mt., $69.2870^{\circ} \mathrm{N} 29.3554^{\circ} \mathrm{E}$, Malaise trap, 1 우 30.VII.11.X.2007, leg. A. Humala. Russia.

DISTRIBUTION. Known from Czech Republic, new for

Mesochorus acutus Schwenke, 1999 stat.rev.

MATERIAL. Karelia, Kpor: Ladozero, $63.580^{\circ} \mathrm{N} 35.884^{\circ} \mathrm{E}$, spruce forest, 1 오 28.VI.2010, leg. A. Humala.

DISTRIBUTION. Known from Central Europe, reported for Karelia [Riedel, Humala, 2016] as M. suomiensis.

REMARK. This species was synonymized with $M$. suomiensis Schwenke, 1999 by Jussila [2011]. We have compared the types of both species and therefore suggest that these taxa are different.

Mesochorus atriventris Cresson, 1872

MATERIAL. Karelia, Kon: Kivach Nature reserve, $62.265^{\circ} \mathrm{N}$ $33.985^{\circ} \mathrm{E}$, light trap, 2 우 $15-18 . X .1990$, leg. A. Humala; Murmansk Area, Lim: Laplandskiy Nature reserve, $67.6513^{\circ} \mathrm{N} 32.5985^{\circ} \mathrm{E}$, spruce forest, Malaise trap, 1우 28.V.-20.IX.2014, leg. A. Humala; Murmansk Area, Lps: Pasvik Nature reserve, Varlam i., pine forest, $69.1376^{\circ} \mathrm{N}$ 29.2616 ${ }^{\circ}$, Malaise trap, 1 ㅇ 10.VII.-3.VIII.2007, leg. A. Humala.

DISTRIBUTION. Known from Karelia [Riedel, Humala, 2016], new for Murmansk Area.

Mesochorus boreomontanus Schwenke, 1999

MATERIAL. Karelia, Kon: Malaya Gomsel'ga, $2 \mathrm{~km} \mathrm{E,}$ $62.05839^{\circ} \mathrm{N} 33.9938^{\circ} \mathrm{E}$, cutting area, 1 우 5.VI.2013, leg. A. Humala.

DISTRIBUTION. Known from Northern and Central Europe, new for Russia. 
Mesochorus cacuminis Schwenke, 1999

MATERIAL. Karelia, Kon: $1 \mathrm{~km}$ S Konchezero, $62.1078^{\circ} \mathrm{N}$ $33.9941^{\circ} \mathrm{E}$, cutting area, yellow pan trap, 1 ㅇ 4-6.VI.2013, leg. A. Humala. Russia.

DISTRIBUTION. Known from Central Europe, new for

Mesochorus cinctus Schwenke, 1999

MATERIAL. Karelia, Kon: Sampo mt., $62.038^{\circ} \mathrm{N} 34.097^{\circ} \mathrm{E}$, pine forest, $1+$ 15.VII.2002, leg. A. Humala.

DISTRIBUTION. Known from Italy, new for Russia.

Mesochorus curvulus Thomson, 1886

MATERIAL. Karelia, $K l: 5 \mathrm{~km} \mathrm{~N}$ Impilahti, $61.7889^{\circ} \mathrm{N}$ $30.8654^{\circ} \mathrm{E}$, meadow, 1 \% 16.VIII.2008, leg. A. Humala.

DISTRIBUTION. Holarctic and Neotropic regions, known from Russia [Meyer, 1929], new for Karelia.

\section{Mesochorus declinans Habermehl, 1922}

MATERIAL. Murmansk Area, Lim: Laplandskiy Nature reserve, $67.6513^{\circ} \mathrm{N} 32.5985^{\circ} \mathrm{E}$, spruce forest, Malaise trap, $1+28$.V.20.IX.2014, leg. A. Humala.

DISTRIBUTION. Known from Russia including Murmansk Area [Riedel, Humala, 2016].

\section{Mesochorus diluvius Schwenke, 1999}

MATERIAL. Karelia, Kton: Bostilovo $7 \mathrm{~km} \mathrm{~S}, 62.15^{\circ} \mathrm{N} 36.78^{\circ} \mathrm{E}$, mixed forest, $1+10$.VIII.2003, leg. A. Humala; Karelia, Kk: 2 km SW of Gridino, $65.9081^{\circ} \mathrm{N} 34.6304^{\circ} \mathrm{E}$, spruce forest, Malaise trap, 1 ㅇ 4.VII.-9.VIII.2007, leg. A. Humala.

DISTRIBUTION. Western Palaearctic region, known from Karelia [Riedel, Humala, 2016].

Mesochorus eichhorni Schwenke, 1999

MATERIAL. Karelia, Kpoc: Voloma river, Nesterov mt. $63.72^{\circ} \mathrm{N} 32.50^{\circ} \mathrm{E}$, spruce forest, $10^{\top} 23$. VI.2000, leg. A. Humala.

DISTRIBUTION. Known from Germany, new for Russia.

Mesochorus errabundus Hartig, 1838

MATERIAL. Karelia, Kon: Kivach Nature reserve, $62.28^{\circ} \mathrm{N}$ $34.00^{\circ} \mathrm{E}$, boggy pine forest, 5 우 4 .VII.2001, leg. A. Humala. 2016].

DISTRIBUTION. Known from Karelia [Riedel, Humala,

Mesochorus frondosus Schwenke, 1999

MATERIAL. Murmansk Area, Lps: Pasvik Nature reserve, Varlam i., $69.1376^{\circ} \mathrm{N} 29.2616^{\circ} \mathrm{E}$, Malaise trap, $1910 . \mathrm{VII}$. 3.VIII.2007, leg. A. Humala.

DISTRIBUTION. Known from Germany and Russian Karelia [Riedel, Humala, 2016], new for Murmansk Area.

Mesochorus fulgurator Horstmann, 2006

MATERIAL. Karelia, Kon: Kivach Nature reserve, $62.265^{\circ} \mathrm{N}$ $33.985^{\circ} \mathrm{E}$, light trap, 1 ㅇ 20-21.VIII.1990, leg. A. Humala.

DISTRIBUTION. Northern and Central Europe, known from Karelia [Riedel, Humala, 2016].

Mesochorus horstmanni Schwenke, 1999

MATERIAL. Finland, $K b$ : Ilomantsi, Pirhu, $62.97^{\circ} \mathrm{N} 31.40^{\circ} \mathrm{E}$, $1 \sigma^{\top}$ 12.VII.1993, leg. A. Humala. land.

DISTRIBUTION. Known from Germany, new for Fin-

Mesochorus hortensis Schwenke, 1999

MATERIAL. Murmansk Area, Lim: Laplandskiy Nature reserve, El'yavr lake, $67.6592^{\circ} \mathrm{N} 32.6417^{\circ} \mathrm{E}$, Malaise trap, 3 우 26.VIII.-22.IX.2014, leg. A. Humala.

DISTRIBUTION. Central Europe, new for Russia.
Mesochorus lacassus Schwenke, 1999

MATERIAL: Murmansk Area, Lim: Laplandskiy Nature reserve, $67.6513^{\circ} \mathrm{N} 32.5985^{\circ} \mathrm{E}$, spruce forest, Malaise trap, 1928 .V.20.IX.2014, leg. A. Humala.

DISTRIBUTION: Known from Italy, new for Russia.

Mesochorus lapponicus Thomson, 1885

MATERIAL. Karelia, Kpor: Myagostrov i., $64.32^{\circ} \mathrm{N} 35.93^{\circ} \mathrm{E}$, seashore, 1 우 14.VIII.2002, leg. A. Humala.

DISTRIBUTION. Northern and Central Europe, new for Russia.

\section{Mesochorus laricis Hartig, 1838}

MATERIAL. Karelia, Kpoc: Zhiloy i., $64.94^{\circ} \mathrm{N} 35.23^{\circ} \mathrm{E}$, supralittoral zone, 1 울 22.VII.2001, leg. A. Humala; Karelia, Kpor: Myagostrov i., $64.32^{\circ} \mathrm{N} 35.93^{\circ} \mathrm{E}$, seashore, $10^{\top} 14$.VIII.2002, leg. A. Humala.

DISTRIBUTION. Western Palaearctic region, known from Karelia [Riedel, Humala, 2016].

Mesochorus perticatus Schwenke, 1999

MATERIAL. Karelia, Kton: Bostilovo, $62.1509^{\circ} \mathrm{N} 36.7816^{\circ} \mathrm{E}$, mixed forest, $1+$ 10.VIII.2003, leg. A. Humala; Karelia, Kpoc: Zhiloy i., $64.94^{\circ} \mathrm{N} 35.23^{\circ} \mathrm{E}$, supralittoral zone, 1 우 22 .VII.2001, leg. A. Humala; Murmansk Area, Lps: Pasvik Nature reserve, Glukhaya Plotina, $69.3653^{\circ} \mathrm{N} 29.7484^{\circ} \mathrm{E}$, yellow pan trap, 1 ㅇ $2-4$.VIII.2011, leg. A. Humala.

DISTRIBUTION. Known from Karelia [Riedel, Humala, 2016], new for Murmansk Area.

Mesochorus pictilis Holmgren, 1860

MATERIAL. Karelia, Kpor: Ladozero, $63.580^{\circ} \mathrm{N} 35.884^{\circ} \mathrm{E}$, spruce forest, $10^{7}$ 28.VI.2010, leg. A. Humala; Murmansk Area, Lim: Laplandskiy Nature reserve, $67.6513^{\circ} \mathrm{N} 32.5985^{\circ} \mathrm{E}$, spruce forest, Malaise trap, 19 28.V.-20.IX.2014, leg. A. Humala.

DISTRIBUTION. Known from Russia [Meyer, 1935, Riedel, Humala, 2016], new for Murmansk Area.

Mesochorus politus Gravenhorst, 1829

MATERIAL. Karelia, Kpor: Myagostrov i., $64.32^{\circ} \mathrm{N} 35.93^{\circ} \mathrm{E}$, seashore, $10^{\top}$ 14.VIII.2002, leg. A. Humala; Karelia, Kpor: Perkhludy, Yuzhnyi i., $64.32^{\circ} \mathrm{N} 36.48^{\circ} \mathrm{E}$, seashore, $10^{7} 16$.VIII.2002, leg. A. Humala.

DISTRIBUTION. Palearctic and Oriental regions, known from Russia [Meyer, 1935, Hellén, 1937, Suh et al., 1997] including Karelia [Riedel, Humala, 2016].

Mesochorus punctipleuris Thomson, 1886

MATERIAL. Murmansk Area, Lps: Pasvik Nature reserve, Varlam i., $69.1376^{\circ} \mathrm{N} 29.2616^{\circ} \mathrm{E}$, Malaise trap, 2 우 $10 . \mathrm{VII} .-$ 3.VIII.2007, leg. A. Humala.

DISTRIBUTION. Widespread in the Holarctic region, known from Russia.

Mesochorus scandinavicus Schwenke, 1999

MATERIAL. Karelia, Kon: Sampo mt., $62.038^{\circ} \mathrm{N} 34.097^{\circ} \mathrm{E}$, pine forest, $10^{7}$ 15.VII.2002, leg. A. Humala.

DISTRIBUTION. Known from Sweden, new for Russia.

Mesochorus semirufus Holmgren, 1860

MATERIAL. Murmansk Area, Lps: Pasvik Nature Reserve, Kalkupya mt., $69.2879^{\circ} \mathrm{N} 29.3554^{\circ} \mathrm{E}$, Malaise trap, 1 ㅇ 30.VII.11.X.2007, leg. A. Humala.

DISTRIBUTION. Widespread in the Palaearctic and Oriental region, known from Karelia [Riedel, Humala, 2016].

Mesochorus skaneus Schwenke, 1999

MATERIAL. Karelia, Kpor: Ladozero, $63.580^{\circ} \mathrm{N} 35.884^{\circ} \mathrm{E}$, spruce forest, $1+28$.VI.2010, leg. A. Humala; Finland, $K b$ : Syväjärvi, $63.03^{\circ} \mathrm{N}$ 
31.35ํㅡ, Malaise trap, 1 우 31.VIII.-4.IX.1990, leg. Tietäväinen. DISTRIBUTION. Known from Finland and Sweden, new for Russia.

\section{Mesochorus tenuiscapus Thomson, 1886}

MATERIAL. Karelia, Kpoc: Voloma river, Nesterov mt., $63.72^{\circ} \mathrm{N} 32.50^{\circ} \mathrm{E}$, spruce forest, $10^{\top} 23$. VI.2000, leg. A. Humala.

DISTRIBUTION. Europe, new for Russia.

Mesochorus testaceus Gravenhorst, 1829

MATERIAL. Karelia, Kol: Gizhino, $61.00^{\circ} \mathrm{N} 33.80^{\circ} \mathrm{E}$, birch forest, Malaise trap, 1우 4-6.VII.2008, leg. A. Humala; Karelia, Kon: Turastamozevo surr., $62.5603^{\circ} \mathrm{N} 34.7163^{\circ} \mathrm{E}$, pine forest, Malaise trap, $10^{7}$ 21.VII.-22.VIII.2012, leg. A. Humala.

DISTRIBUTION. Widespread in the Palaearctic region, known from Russia [Becker, 1857, Szépligeti, 1914] including Karelia [Riedel, Humala, 2016].

Mesochorus tachypus Holmgren, 1860

MATERIAL. Karelia, Kpoc: Zhiloy i., $64.94^{\circ} \mathrm{N} 35.23^{\circ} \mathrm{E}$, supralittoral zone, 2 우 22.VII.2001, leg. A. Humala.

DISTRIBUTION. Western Palaearctic region, known from Karelia [Riedel, Humala, 2016].

REMARK. This species has been called " $M$. tetricus Holmgren, 1860" in the revision by Schwenke [1999: 77], but Vikberg and Vårdal [2017] have clarified and corrected the synonymy of some Mesochorus taxa described by Holmgren, including this species.

\section{Mesochorus tetricoides sp.n.}

Figs 1-2.

MATERIAL. Holotypus: (†) RUSSIA, Karelia, Kon: Kivach Nature reserve, $62.265^{\circ} \mathrm{N} 33.985^{\circ} \mathrm{E}$, light trap, A. Humala, 58.X.1990 (ZSM).
DESCRIPTION. Female: Body length $3.3 \mathrm{~mm}$. Flagella with 29 segments; first flagellomere length $6.8 \mathrm{x}$ width and $0.7 \mathrm{x}$ eye length; second flagellomere length $4.3 \mathrm{x}$ width; preapical flagellomeres c.2x longer than wide. Temples moderately and roundly narrowed behind eyes, length $0.8 x$ eye width. Distance of lateral ocelli to eyes c.1.1x ocellar diameter. Face wide, width $1.1 \mathrm{x}$ length of clypeus + face and $0.95 \mathrm{x}$ eye length, sides slightly convergent, with fine punctures, shining. Ventral half of facial orbits and malar spaces widely striate. Frons and occiput almost smooth. Malar space length c. $0.8 \mathrm{x}$ width of mandibular base. Mandibles with two equal teeth.

Mesoscutum with very superficial punctures, smoothened frontally and finely granulate caudally. Mesopleura with very fine and scattered hair-punctures. Area superomedia c. $2 \mathrm{x}$ longer than wide, costulae frontal to its middle; area petiolaris about as long as wide, finely rugulose. Hind femora length 4.6x height; hind claws with short basal teeth. Areolet sessile, second recurrent veins proximal to their middle; nervuli slightly postfurcal (by $0.5-1 \mathrm{x}$ their diameter).

First tergite length $2.4 \mathrm{x}$ width, postpetiolus smooth; second tergite length 1.0x width; thyridia transverse-oval. Ovipositor sheaths thick, stab-shaped, completely pilose, length $6.7 \mathrm{x}$ width and $1.3 \mathrm{x}$ length of hind metatarsi.

Color: Black. Palps, mandibles, malar spaces, ventral half of facial orbits and clypeus apically yellowish; vertical orbits opposite to lateral ocelli with indistinct reddish spots. Flagella blackish, basal three flagellomeres pale brownish ventrally. Tegulae yellowish, mesosoma otherwise blackish. Metasoma blackish; tergites 2-7 with very narrow pale hind margins. Legs brownish-red; all coxae brownish; hind tibiae yellowish, basally and in apical 0.1 diffusely brownish; hind tarsi brown. Pterostigma ochre-yellow.

Male unknown.
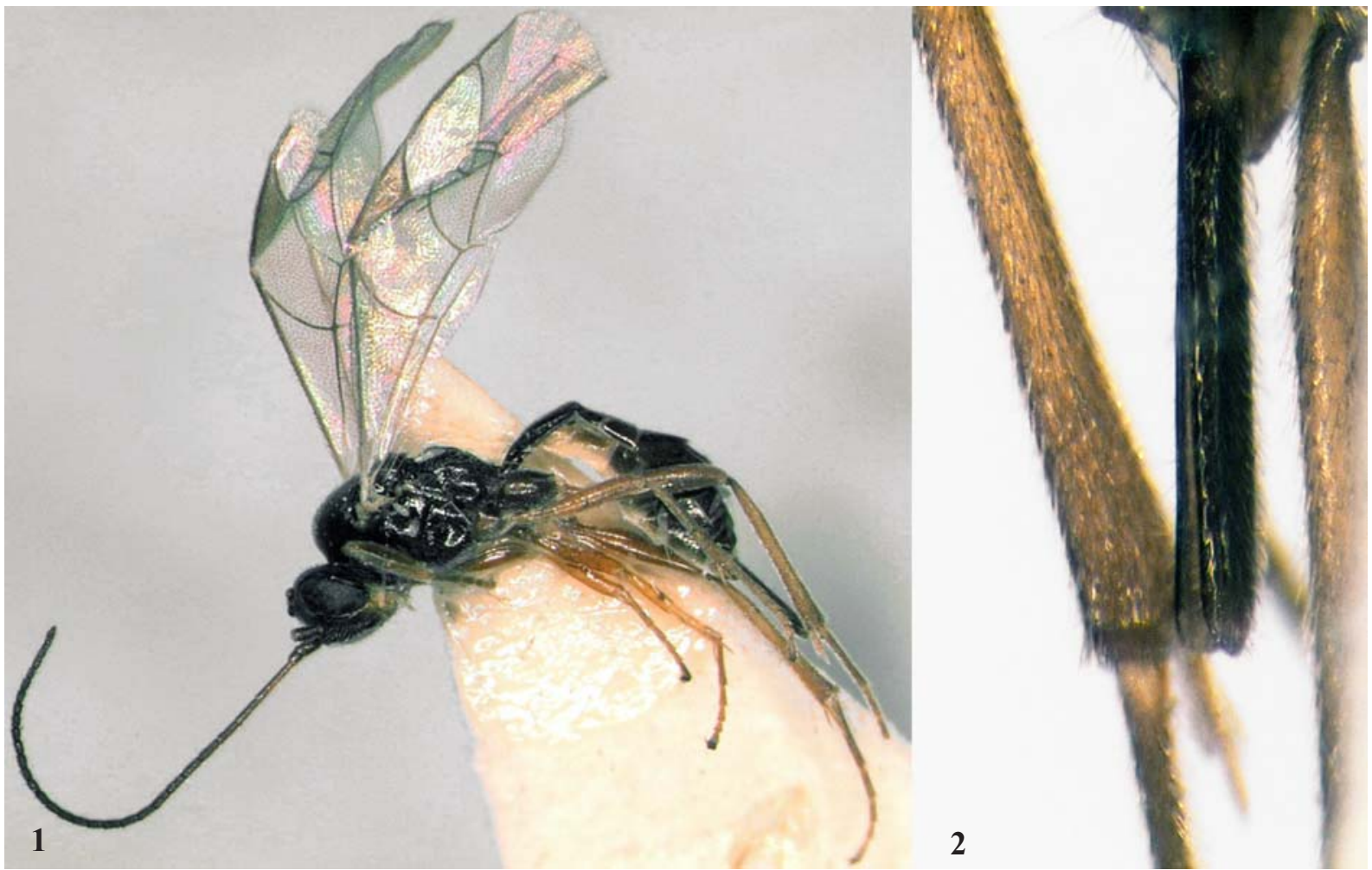

Figs 1-2. Mesochorus tetricoides sp.n.: 1 - general habitus; 2 - ovipositor sheaths, lateral view. Рис. 1-2. Mesochorus tetricoides sp.n.: 1 - общий вид; 2 - ножны яйцеклада, вид сбоку. 
TAXONOMIC REMARKS. This new taxon belongs to the angustatus group of Mesochorus and is closely related to M. tachypus Holmgren, 1860 (tetricus Holmgren, 1860 sensu Schwenke, 1999). It differs by its smaller size, completely blackish mesosoma, paler pterostigma and narrow apical obfuscation of hind tibiae. It can be differentiated from the newly described M. skanensis Vikberg, 2017 by its color pattern (mainly blackish color of mesosoma and metasoma) and straight ovipositor sheaths. It can be differentiated from species of the nigripes group, especially $M$. punctipleuris, by the scattered puncture of mesopleura, longer and stouter ovipositor sheaths, and almost completely blackish metasoma.

\section{Mesochorus vittator (Zetterstedt, 1838)}

MATERIAL. Karelia, Kton: $6 \mathrm{~km}$ E Muromskoe lake, $61.487^{\circ} \mathrm{N}$ $36.540^{\circ} \mathrm{E}$, spruce forest, $10^{7} 14 . \mathrm{VI} .2003$, leg. A. Humala; Karelia, $K p$ : Chumbozero, $61.8412^{\circ} \mathrm{N} 37.7534^{\circ} \mathrm{E}$, mixed forest, Malaise trap, 1 + 20-25.VI.2009, leg. A. Humala; Murmansk Area, Lps: Pasvik Nature reserve, Varlam i., $69.1376^{\circ} \mathrm{N} 29.2616^{\circ} \mathrm{E}$, Malaise trap, $20^{7} 0^{\top}$ 10.VII.-3.VIII.2007, leg. A. Humala.

DISTRIBUTION. Widespread in the Holarctic and Neotropical regions, known from Russia [Meyer, 1929, 1935, Uchida, 1933, 1935] including Murmansk Area [Woldstedt, 1873] and Karelia [Riedel, Humala, 2016].

\section{Mesochorus vitticollis Holmgren, 1860}

MATERIAL. Karelia, Kol: Gizhino, $61.00^{\circ} \mathrm{N} 33.80^{\circ} \mathrm{E}$ birch forest, Malaise trap, 19 4-6.VII.2008, leg. A. Humala.

DISTRIBUTION. Widespread in the Western Palaearctic region, known from Russia [Meyer, 1935] including Karelia [Krogerus, 1938, Riedel, Humala, 2016]

ACKNOWLEDGEMENTS. The second author conducted this study under the state assignment to the Forest Research Institute KarRC RAS (No. 0220-2015-00014).

\section{References}

Becker A. 1857. Naturhistorischer Bericht aus der Umgebung von Serapta vom Jahre 1855 und einige Bemerkungen über das Tödten und Fangen der Insekten // Bulletin de la Société Impériale des Naturalistes de Moscou. T.30. P.250-272.

Heikinheimo O., Raatikainen M. 1971. The recording of locations of biological finds in Finland // Annales Entomologici Fennici. Vol.37. No.1a. P.1-27.

Hellén W. 1937. Für die Fauna Finnlands neue Ichneumoniden (Hym.) III. // Notulae Entomologicae. Vol.17. S.52-56.

Hellén W. 1942. Zur Ichneumonidenfauna Finnlands (Hym.) III. // Notulae Entomologicae. Vol.22. S.76-87.

Jussila R. 2011. Lisäyksiä Suomen ja muun länsipalearktisen alueen Mesochorus-lajistoon (Hymenoptera, Ichneumonidae, Mesochorinae) // Sahlbergia. Vol.17. No.1. S.32-55.
Kravchenko A.V., Kuznetsov O.L. 2001. [Peculiarities of biogeographical provinces of the Republic of Karelia on the basis of analysis of vascular plant flora] // Trudy Karel'skogo nauchnogo tsentra RAN. Biogeographia Karelii. No.2. P.59-64 [in Russian, with English summary].

Krogerus R. 1938. Parasitsteklar från torvmakerna i Kuusamoområdet // Notulae Entomologicae. Vol.18. S.105-108.

Meyer N.F. 1929. Schlupfwespen, die in Russland in den Jahren 1891-1926 aus Schädlingen gezogen sind (Vortsetzung) // Izvestiya Otdela Prikladnoy Entomologii. Vol.4. P.231-248 [in Russian].

Meyer N.F. 1935. [Parasitic Hymenoptera of the family Ichneumonidae of the USSR and adjacent countries. Part 4. Ophioninae]. Moscow-Leningrad: Akademia Nauk SSSR Press. 16. Vol.4. P.15-35 [in Russian].

Riedel M. 2015. Revision of the European species of the genus Astiphromma Förster, 1869 (Hymenoptera, Ichneumonidae, Mesochorinae) // Spixiana. Vol.38. P.85-132.

Riedel M., Humala A. 2016. Faunistic notes on the Mesochorinae (Hymenoptera, Ichneumonidae) of Northwestern Russia, with descriptions of the males of Astiphromma flavoventrale Riedel and A. flagellator Riedel // Russian Entomological Journal. Vol.25. No.1. P.65-69.

Roman A. 1931. Entomologische Ergebnisse der schwedischen Kamtschatka-Expedition 1920-1922. 33. Ichneumonidae, Subfamilien Pimplinae und Tryphoninae // Arkiv för Zoologi. Vol.23A. No.6. S.1-32.

Schwenke W. 1999. Revision der europäischen Mesochorinae (Hymenoptera, Ichneumonoidea, Ichneumonidae) // Spixiana, Supplement. Vol.26. S.1-124.

Suh K.I., Lee J.W., Choi W.Y 1997. A systematic study of the Mesochorinae (Hymenoptera: Ichneumonidae) from the eastern Palearctic region 1. A review of the genus Mesochorus // Entomological Research Bulletin. Vol.23. P.1-28.

Szépligeti G. 1914. Ichneumoniden aus der Sammlung des ungarischen National Museums // Annales Musei Nationalis Hungarici. Vol.12. S.414-434

Söderman G., Várkonyi G., Koponen M. 2010. Parasitoid wasps. Hymenoptera: Parasitica. // P. Rassi, E. Hyvärinen, A., Juslén et I. Mannerkoski (eds.). The 2010 Red List of Finnish Species. Ministry of the Environment. Edita Ltd. Helsinki 2010. 685 pp.

Uchida T. 1933. Beiträge zur Systematik der Tribus Mesochorini Japans (Hym. Ichneumonidae) // Insecta Matsumurana. Vol.8. S.51-63.

Uchida T. 1935. Beiträge zur Kenntnis der Ichneumonidenfauna der Kurilen // Insecta Matsumurana. Vol.9. S.108-122.

Vikberg V., Vårdal H. 2017. Taxonomy of some European species of Mesochorus, including three new species from Finland and Sweden (Hymenoptera: Ichneumonidae: Mesochorinae) // walbum. Vol.20. P.3-42.

Woldstedt F.W. 1873. Materialier till en Ichneumonologia Fennica // Aftryck ur Finska Vetenskapssocietetens. Bidrag till Kännedom af Finnlands Natur och Folk. H.21. Helsingfors. S.61-92.

Yu D.S.K., van Achterberg C., Horstmann K. 2016. Taxapad 2016, Ichneumonoidea 2015. Database on flash-drive. www.taxapad.com, Nepean, Ontario, Canada. 